\title{
DIRECT ORTHOGONAL SIGNAL CORRECTION-PARTIAL LEAST SQUARES FOR SIMULTANEOUS SPECTROPHOTOMETRIC DETERMINATION OF SULFAMETHOXAZOLE AND TRIMETHOPRIM IN PHARMACEUTICAL FORMULATION AND SYNTHETIC SAMPLES
}

\author{
MOHAMMAD GOODARZI I,2, * PARVIZ SHAHBAZIKHAH ${ }^{3}$, MAHMOUD REZA SOHRABI $^{4}$ \\ MOZHGAN FATHABADI ${ }^{4}$, SAREZOO HASSAN NOURI ${ }^{4}$
}

\author{
${ }^{\prime}$ Department of Chemistry, Faculty of Science, Azad University of Arak, Arak, Iran \\ ${ }^{2}$ Young Researches Club, Azad University, Arak, Iran \\ ${ }^{3}$ Department of Chemistry, Sharif University of Technology, Tehran, Iran \\ ${ }^{4}$ Department of chemistry, Azad University-North Tehran Branch, Tehran, Iran \\ ${ }^{5}$ Young Researches Club, Islamic Azad University, North Tehran Branch, Tehran, Iran
}

(Received: September 22, 2008 - Accepted: June 9, 2009)

\begin{abstract}
This work describes a very simple, novel and selective spectrophotmetric method for simultaneous determination of sulfamethoxazole and trimethoprim in pharmaceutical preparation and synthetics samples using direct orthogonal signal correction-partial least squares (DOSC-PLS). The all factors affecting on the sensitivity were optimized and the linear dynamic range for determining of these drugs was found. The PLS modeling was used for the multivariate calibration of the spectrophotometric data and DOSC was used for preprocessing of data matrices and the prediction results of model. The experimental calibration matrix was designed by measuring the absorbance over the range 200-400 nm for 32 samples of 2.0-15.0 and 0.4-6.0 $\mu \mathrm{g} \mathrm{mL}^{-1}$ of sulfamethoxazole and trimethoprim, respectively. The root mean square error of prediction for sulfamethoxazole and trimethoprim using DOSC-PLS and PLS were $0.02212,0.02363$ and 0.17014 , $0.01660, \mu \mathrm{g} \mathrm{mL} \mathrm{m}^{-1}$ respectively. Application of the method to synthetic samples of standard solutions and in pharmaceutical formulation was performed and satisfactory results were obtained.
\end{abstract}

Keywords: Sulfamethoxazole, Trimethoprim, Spectrophotometric, PLS, DOSC-PLS.

\section{INTRODUCTION}

Sulfonamides are highly effective chemotherapeutic drugs well known as antibacterial agents widely used in medicine and veterinary practices. Many pharmaceutical products are now available which contain sulfonamides combinations with another drug that its function is increasing the antibiotic effectively. Someofthese commercial formulationsinclude: sulfaquinoxalineand prymethamine, sulfadiazine and trimethoprim (TMP), sulfamethoxazole (SMX) and TMP, sulphamethoxypyridazine (SMP) and TMP, etc. Sulfamethoxazole (SMX) or 5-methyl-3-sulfanylamidoisoxazole is a chemotherapeutics agent widely used as antibacterial drug. Its individual determination has been carried out by spectrophotometry ${ }^{1,2}$ and by fluorimetry $y^{3,4}$. HPLC has also been proposed for determination of SMX and its main metabolites in human plasma and urine ${ }^{5}$. The pharmaceuticals containing sulphonamides consist only of one drug or one sulphonamide associated with another drug, which increases the power of the sulphonamide. So, SMX is usually combined with trimethoprim (TMP) in a fixed proportion 5:1, respectively, this association being called cotrimoxazol. TMP or 2, 4-diamino-5-(3, 4, 5-trimethoxybenzyl) - pyrimidine is also a bacteriostatic drug. Its determination in pharmaceutical preparations has been usually carried out by spectrophotometric methods ${ }^{6,7}$ and sometimes by electro analytical methods ${ }^{8,9}$. Liquid chromatography ${ }^{10,12}$ and gas chromatography ${ }^{13}$ have also been proposed for its individual determination or together with its major metabolites of oxidation in different matrices. The simultaneous determination of both analytes has been usually carried out by spectrophotometric methods with multi component analysis based on the use of second derivative and diode-array detection ${ }^{14}$, first derivative and spectra ratio $^{15}$, bivariate calibration spectrophotometrics ${ }^{16}$, high-performance liquid chromatography (HPLC $)^{17}$. However, these methods are generally complex in nature and need expensive instruments and ultra pure solvents. In other hand, analysis of the clinical samples demands simple and fast analytical methods and therefore, finding an alternative analytical procedure or technique is crucial. Spectrophotometry combined with chemometrics methods will be a simple analytical method for quantitative analysis ${ }^{18,19}$. Chemometrics is a field of science that studied the application of statistical and mathematical methods in chemistry. One of the chemometrics methods is multivariate calibration technique. Multivariate calibration is a collection of powerful mathematical tools that can be applied to resolve complexity in chemical analysis. It is useful in spectral analyses because the simultaneous inclusion of multiple spectral intensities can greatly improve the precision and applicability of quantitative spectral analysis of multicomponent mixtures that can not be resolved by conventional spectrometry. In recent years multivariate calibration has become an important tool in resolution of mixtures of components in many different fields including biomedical ${ }^{20,21}$ environmental ${ }^{22,23}$ and drug analysis ${ }^{24,25}$. This paper describes an analytical methodology for simultaneous determination of Sulfamethoxazole and Trimethoprim using spectrophotometric method with preprocessing by direct orthogonal signal correction (DOSC). Data collected from complicated samples or an uncomplicated process contains variation from many sources and of several types. Preprocessing methods can be applied in such situations to enhance the relevant information to make resulting models simpler and easier to interpret. Orthogonal signal correction (OSC) filters was developed to remove strong structured (i.e. systematic) variation in $\mathbf{X}$ that is not correlated to $\mathbf{Y}$. That is, they remove structured $\mathrm{Y}$-orthogonal variation from $\mathbf{X}$, in such a way that the filter can be applied to future data. The OSC filters need information about $\mathbf{Y}$. If no $\mathbf{Y}$ exists, it may be possible to create it by adding dummy variables $(1 / 0)$. In a similar fashion, the OSC methods can also be used to remove $\mathrm{X}$-orthogonal variation in $\mathbf{Y}$. Wold et al ${ }^{26}$ published the original work on OSC. Two different approaches are used to construct OSC filters, they have divided to the indirect (original approach) and the direct approach based on the way they estimate the OSC component. Since the indirect approaches had some problems concerning the orthogonality towards $\mathbf{Y}$, non-optimal amount of variance removed from $\mathbf{X}$, a new direct OSC algorithm (DOSC) was utilized (27). This method is developed using only simple least squares steps. DOSC determines the OSC components in a more straightforward way and they are guaranteed to be orthogonal to $\mathbf{Y}$. No 'internal' regression model is needed, so there are no associated overfit problems. The direct OSC approach also offers the possibility of creating specific OSC filters. The aim of this work is to offer direct orthogonal signal correction method to resolve the mixture of sulfamethoxazole and trimethoprim in synthetic and real samples.

Theory

Westerhuis et al..$^{27}$ introduced direct orthogonal signal correction. The DOSC algorithm calculates directions in $\mathbf{X}$ that are orthogonal to $\mathbf{Y}$ and account for the largest variance of $\mathbf{X}$. These directions are obtained by only using least squares steps.

The first step of DOSC is to decompose $\mathbf{Y}$ in two orthogonal parts. In this way, $\mathbf{Y}$ decomposes in to $\hat{\mathbf{Y}}$, the projection of $\mathbf{Y}$ onto $\mathbf{X}$, and $\mathbf{F}$, the residual part that is unrelated to $\mathbf{X}$ (orthogonal to $\mathbf{X}$ ):

$$
\text { 1. } \mathbf{Y}=\mathbf{P}_{\mathrm{X}} \mathbf{Y}+\mathbf{A}_{\mathrm{X}} \mathbf{Y}=\hat{\mathbf{Y}}+\mathbf{F}
$$

Next, $\mathbf{X}$ is decomposed into two orthogonal parts, one part that has the same range as $\hat{\mathbf{Y}}$ and another part that is orthogonal to it.

2. $\mathbf{X}=\mathbf{P}_{\hat{\mathbf{Y}}} \mathbf{X}+\mathbf{A}_{\hat{\mathbf{Y}}} \mathbf{X}$ 


\section{3. $\mathbf{A}_{\hat{\mathbf{Y}}} \mathbf{X}=\mathbf{X}-\mathbf{P}_{\hat{\mathrm{Y}}} \mathbf{X}$}

Therefore, the columns of $\mathbf{A}_{\hat{\mathbf{Y}}} \mathbf{X}$ span a subspace of $\mathbf{X}$ that is orthogonal both to $\hat{\mathbf{Y}}$ and to $\mathbf{Y}=\hat{\mathbf{Y}}+\mathbf{F}$, since $\mathbf{F}$ is also orthogonal to $\mathbf{X}$.

With having this orthogonal subspace $\mathbf{A}_{\hat{\mathbf{Y}}} \mathbf{X}$, principle component analysis (PCA) is applied to it in order to find the principle component $\mathbf{T}$ corresponding to the largest singular value. If more DOSC components are necessary, more principal components can be obtained in this step. $\mathbf{T}$ is a basis for the onedimensional subspace that accounts for maximum variance of $\mathbf{A}_{\hat{\mathbf{Y}}} \mathbf{X}$. This then is the sought for one-dimensional subspace of $\mathbf{X}$ that is orthogonal to $\mathbf{Y}$ and accounts for the maximum possible variance of $\mathbf{X}$. The direction $\mathbf{T}$ can be expressed as linear combination of $\mathbf{X}$ :

\section{4. $T=X W$}

where $\mathbf{W}$ is weight matrix for determination of DOSC components and equal to $\mathbf{X}^{+} \mathbf{T}$, where $\mathbf{X}^{+}$is Moore-Penrose generalized inverse of original matrix $\mathbf{X}$. The large-variance zero-correlation part of $\mathbf{X}$ that we do not use in subsequent regression modeling is removed from the data:

5. $\mathbf{X}_{\text {DOSC }}=\mathbf{X}-\mathbf{T P}^{\mathrm{T}}$

where $\mathbf{P}$ is loading and can be expressed as:

6. $\mathbf{P}=\mathbf{X}^{\mathrm{T}} \mathbf{T}\left(\mathbf{T}^{\mathrm{T}} \mathbf{T}\right)^{-1}$

Having weights $\mathbf{W}$ and loading $\mathbf{P}$, one can directly correct new spectral data $\mathbf{X}_{\text {new }}$ as follows:

7. $\mathbf{X}_{\text {new Dosc }}=\mathbf{X n e w}-\mathbf{X n e w} \mathbf{W P}^{\mathrm{T}}$ $\mathbf{Y}_{\text {new }}{ }^{27}$.

Now $\mathbf{X}_{\text {new,DosC }}$ can be used in calibration model instead of $\mathbf{X}_{\text {new }}$ to predict

\section{EXPERIMENTAL}

\section{Reagents}

All chemicals were reagent grade chemicals. Sulfonamides standard solutions were prepared in ethanol, with sulfamethoxazole $(98 \% \mathrm{w} / \mathrm{w})$, trimethoprim $(95 \% \mathrm{w} / \mathrm{w})$. These standards were obtained from Fluka. Extra pure ethanol (Eth,OH, Fluka) was used as received. Stock standard solutions of sulfamethoxazole and trimethoprim, $1000 \mu \mathrm{g} \mathrm{mL}^{-1}$, were prepared by dissolving appropriate amount of solutes in ethanol. Working solutions of lower concentrations were prepared by proper dilution with ethanol from the stock standard solutions.

\section{Procedures}

General procedure

An experimental design was used to maximize statistically the information content in the spectra ${ }^{28}$. A training set of 26 samples was taken (Table 1). The concentrations of sulfamethoxazole and trimethoprim were varied between 2.0-15.0 and 0.4-6.0 $\mu \mathrm{g} \mathrm{mL}^{-1}$, respectively. Samples were prepared in $10 \mathrm{ml}$ volumetric flasks by adding between $2.0-15.0(\mu \mathrm{g} / \mathrm{ml})$ sulfamethoxazole and 0.4-6.0 $\mu \mathrm{g} \mathrm{mL}^{-1}$ trimethoprim, $1.0 \mathrm{ml}$ of ethanol, $2.5 \mathrm{ml}$ of ammonium buffer solution ( $\mathrm{pH} 10.0)$ and adjusted to the final volume with deionized water. The absorption spectra were recorded between 200 and $400 \mathrm{~nm}$ against blank of ethanol. The spectra region between 200 and $400 \mathrm{~nm}$, which implies working with 200 experimental points per as spectra (as the spectra are digitized each $1.0 \mathrm{~nm}$ ), was selected for analysis, because this is the zone with the maximum spectral information from the mixture components of interest.

Table 1. Concentration data of the different mixtures used in the calibration set for the determination of sulfamethoxazole and trimethoprim $\left(\mu \mathrm{g} \mathrm{mL}^{-1}\right)$

\begin{tabular}{|c|c|c|c|c|c|c|c|c|}
\hline Mixture & Sulfamethoxazole & Trimethoprim & Mixture & Sulfamethoxazole & Trimethoprim & Mixture & Sulfamethoxazole & Trimethoprim \\
\hline M1 & 2.0 & 0.5 & M10 & 8.0 & 6.0 & M19 & 15.0 & 3.0 \\
\hline M2 & 4.0 & 1.0 & M11 & 9.0 & 2.0 & M20 & 15.0 & 5.0 \\
\hline M3 & 5.0 & 1.0 & M12 & 10.0 & 2.5 & M21 & 3.0 & 1.0 \\
\hline M4 & 5.0 & 1.6 & M13 & 10.0 & 4.0 & M22 & 4.0 & 2.0 \\
\hline M5 & 5.0 & 2.0 & M14 & 12.0 & 2.0 & M23 & 6.0 & 1.5 \\
\hline M6 & 6.0 & 1.2 & M15 & 12.0 & 3.0 & M24 & 8.0 & 4.0 \\
\hline M7 & 6.0 & 2.0 & M16 & 12.0 & 4.0 & M25 & 8.0 & 5.0 \\
\hline M8 & 8.0 & 0.4 & M17 & 14.0 & 3.0 & M26 & 9.0 & 3.0 \\
\hline M9 & 8.0 & 2.0 & M18 & 14.0 & 5.0 & & \\
\hline
\end{tabular}

\section{Real sample}

For the analysis, 10 tablets of the pharmaceutical Co-trimoxazole were weighted and ground to fine powder. Then a proportion of powder equivalent to one average tablet weighted and dissolved in ethanol. The solution was filtered and diluted to an appropriate volume with ethanol. Absorbance spectra were recorded.

\section{Linear calibration range}

Individual calibration curves were constructed with several points as absorbance versus sulfamethoxazole and trimethoprim concentrations. For constructing the individual calibration lines, the absorbencies were measured at 269 and $236 \mathrm{~nm}$ against a blank for sulfamethoxazole and trimethoprim, respectively. The linear regression equation for the calibration graph for sulfamethoxazole for the concentration range of $2.0-15.0 \mu \mathrm{g} \mathrm{mL}$ ${ }^{1}$ was $\mathrm{A}=0.1995 \mathrm{C}+0.0165\left(\mathrm{r}^{2}=0.9984\right)$ and for trimethoprim for the concentration range $0.4-6.0 \mu \mathrm{g} \mathrm{mL}^{-1}$ was $\mathrm{A}=0.2945 \mathrm{C}_{\text {trimethoprim }}+0.1096$ $\left(\mathrm{r}^{2}=0.9980\right)$. The detection limits (LOD) for the proposed methods were calculated using the following equation ${ }^{29}$

$$
L O D=\frac{3 s}{k}
$$

where $s$ is the standard deviation of replicate determination values under the same conditions as for the sample analysis in the absence of the analyte and $k$ is the sensitivity, namely the slope of the calibration graph. The limits of detection were 0.03 and $0.07 \mu \mathrm{g} \mathrm{mL}^{-1}$ for sulfamethoxazole and trimethoprim, respectively, and these were calculated according to calibration line characteristics.

\section{Apparatus and software}

UV-vis spectrophotometer BIO-TEK-KONTRON (UVIKON922) equipped with $1.00 \mathrm{~cm}$ path length quartz cells was used for acquisition of spectral data. A Metrohm $692 \mathrm{pH}$-meter furnished with a combined glasssaturated calomel electrode was calibrated with at least two buffer solutions at pH 3.00 and 9.00. The DOSC algorithm was written in MATLAB $6.5^{30}$, using the corresponding toolbox. All programs were run on a personal computer (Pentium IV) with Windows XP operational system. PLS calculus were carried out in the 'PLS Toolbox', version 2.0 (Eigenvectors Company).

\section{RESULTS AND DISCUSSION}

Optimum experimental conditions were studied previously for the mixture determination of the analytes ${ }^{31}$. The effect of $\mathrm{pH}$ on the absorption spectra of SMX and TMP were studied previously at the corresponding maximum absorption wavelength and a value of $\mathrm{pH} 10.0$ were selected based on sensitivity and stability of the analytes ${ }^{16}$. The absorption spectra of SMX and TMP are shown in Figure 1. As this figure shows, there is a clear overlapping of the two spectra. This prevents the simultaneous determination of the sulfamethoxazole and trimethoprim by direct UV-vis absorbance measurements. To overcome this problem a suitable and simple technique, which proposed as a powerful technique for solving these problems and presents a good recovery, is DOSCPLS. It is worth mentioning that for calibration set and prediction set two DOSC components were used for filtering. Evaluation of the prediction errors 
for the validation set reveals that the DOSC treated data give significantly lower RMSEP values than original data. Also, the DOSC-filtered data give much simpler calibration models with fewer components than the ones based on original data. The results imply that the DOSC method indeed removes information from UV-vis data that is not necessary for fitting of the Y-variables.

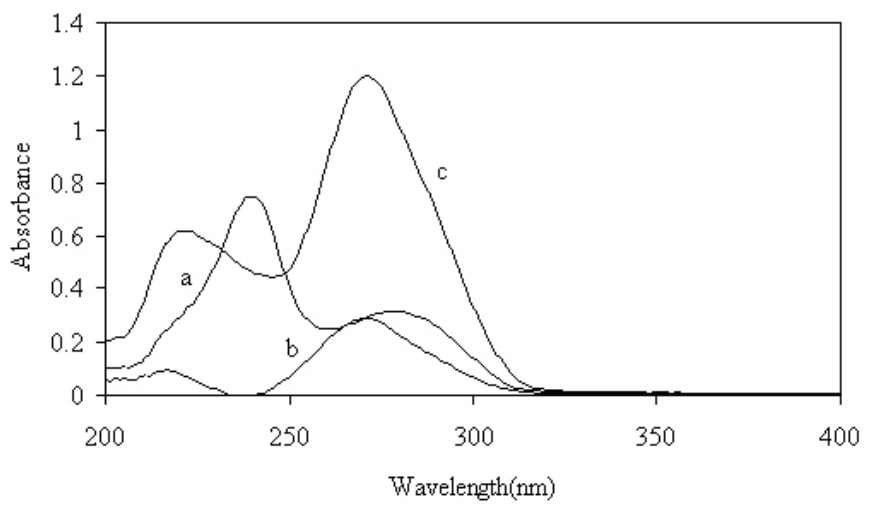

Figure 1. Absorption spectra of (a) trimethoprim, (b) sulfamethoxazole and (c) mixtures of sulfamethoxazole and trimethoprim (Concentration of each components is $\left.6.0 \mu \mathrm{g} \mathrm{mL}^{-1}\right)$.

Calibration and validation

According to Section 3.2.3, the calibration matrix was designed. In Table 1, the compositions of the two mixtures used in the calibration matrices are summarized. For prediction set, six mixtures were prepared (see Table 2). To ensure that the prediction are in the subspace of training set, the score plot of first three principal components was sketched and all the samples are spanned with the training set scores .

Table 2. Composition of synthetic mixtures and predicted values for determination of sulfamethoxazole and trimethoprim $\left(\mu \mathrm{g} \mathrm{mL} L^{-1}\right)$.

\begin{tabular}{|c|c|c|c|c|c|c|c|c|c|}
\hline \multicolumn{2}{|c|}{ Added } & \multicolumn{2}{c|}{ Determined (PLS) } & \multicolumn{2}{c|}{ Recovery (\%) } & \multicolumn{2}{c|}{ Determined (DOSC-PLS) } & \multicolumn{2}{c|}{ Recovery (\%) } \\
\hline $\begin{array}{c}\text { Sulfame- } \\
\text { thoxazole }\end{array}$ & $\begin{array}{c}\text { Trime- } \\
\text { thoprim }\end{array}$ & $\begin{array}{c}\text { Sulfame- } \\
\text { thoxazole }\end{array}$ & $\begin{array}{c}\text { Trime- } \\
\text { thoprim }\end{array}$ & $\begin{array}{c}\text { Sulfame- } \\
\text { thoxazole }\end{array}$ & $\begin{array}{c}\text { Trime- } \\
\text { thoprim }\end{array}$ & $\begin{array}{c}\text { Sulfame- } \\
\text { thoxazole }\end{array}$ & $\begin{array}{c}\text { Trime- } \\
\text { thoprim }\end{array}$ & $\begin{array}{c}\text { Sulfame- } \\
\text { thoxazole }\end{array}$ & $\begin{array}{c}\text { Trime- } \\
\text { thoprim }\end{array}$ \\
\hline 6.0 & 1.5 & 5.94 & 1.53 & 99.05 & 101.5 & 5.99 & 1.50 & 99.96 & 100.0 \\
\hline 8.0 & 5.0 & 7.86 & 4.67 & 98.30 & 93.45 & 8.00 & 4.98 & 100.0 & 99.71 \\
\hline 3.0 & 1.0 & 2.77 & 1.15 & 92.37 & 114.7 & 2.98 & 1.01 & 99.27 & 101.5 \\
\hline 4.0 & 2.0 & 3.91 & 1.88 & 97.91 & 94.18 & 3.97 & 2.02 & 99.35 & 101.3 \\
\hline 8.0 & 4.0 & 8.27 & 4.15 & 103.4 & 103.7 & 7.97 & 4.03 & 99.60 & 100.8 \\
\hline 9.0 & 3.0 & 9.13 & 3.02 & 101.4 & 100.6 & 9.02 & 2.97 & 100.3 & 98.95 \\
\hline
\end{tabular}

Selection of the optimum number of factors in PLS model.

The optimum number of factors (latent variables) to be included in the calibration model was determined by computing the prediction error sum of squares (PRESS) for cross- validated models using a high number of factors (half the number of total standard +1 ), which is defined as follows:

$$
P R E S S=\sum\left(y_{i}-\hat{y}_{i}\right)^{2}
$$

Where $y_{\mathrm{i}}$ is the reference concentration for the $i$ th sample and $y_{\mathrm{i}}$ represents the estimated concentration. The cross-validation method employed was to eliminate only one sample at a time and then PLS calibrate the remaining standard spectra. By using this calibration the concentration of the sample, left out was predicted. This process was repeated until each standard had been left out once. One reasonable choice for the optimum number of factors would be that number which yielded the minimum PRESS. Since there are a finite number of samples in the training set, in many cases the minimum PRESS value causes over fitting for unknown samples that were not included in the model. A solution to this problem has been suggested by Haaland and et al 32 in which the PRESS values for all previous factors are compared to the PRESS value at the minimum. The F-Statistical test can be used to determine the significance of PRESS values greater than the minimum. The maximum number of factors used to calculate the optimum PRESS was selected as 14 and the optimum number of factors obtained by the application of PLS models is summarized in Table 3. In all instances, the number of factors for the first
PRESS values whose F-ratio probability drops below 0.75 was selected as the optimum. In Figure 2, the PRESS obtained by optimizing the calibration matrix of the absorbance data with PLS model is shown. It should be noted that the PLS model was run twice. In the first run, the original spectral data matrix was used without any pre-processing. Results showed that the PRESS values are minimum in the number of factors of 4,3 for sulfamethoxazole and trimethoprim, respectively. In the second run (DOSC-PLS), firstly the original spectral data (calibration and prediction sets) were corrected with DOSC using 2 number of factor. Then the DOSC corrected spectral data was directly used for calibration and prediction. The obtained results show that the PRESS values are minimum in the number of factor of 3 for both of them.

Determination of sulfamethoxazole and trimethoprim in synthetic mixtures

The determination of the sulfamethoxazole and trimethoprim in mixtures by spectrophotometric using multivariate calibration involved constructing model using calibration set. According to Section 3.2.3, the calibration matrix designed. In Table 1, the compositions of the two mixtures used in the calibration matrices are summarized. The predictive ability of method was determined using six two-component sulfamethoxazole and trimethoprim mixtures (their compositions are given in Table 2). The results obtained by applying DOSCPLS algorithm to six synthetic samples are listed in Table 2. Table2 also shows the recovery for prediction series of sulfamethoxazole and trimethoprim mixtures. As can be seen, the recovery was also quite acceptable. The root mean square error of prediction and relative standard error of prediction results are summarized in Table3. The plots of the predicted concentration versus actual values are shown in Figure 3 for sulfamethoxazole and trimethoprim (line equations and $R^{2}$ values are also shown). 

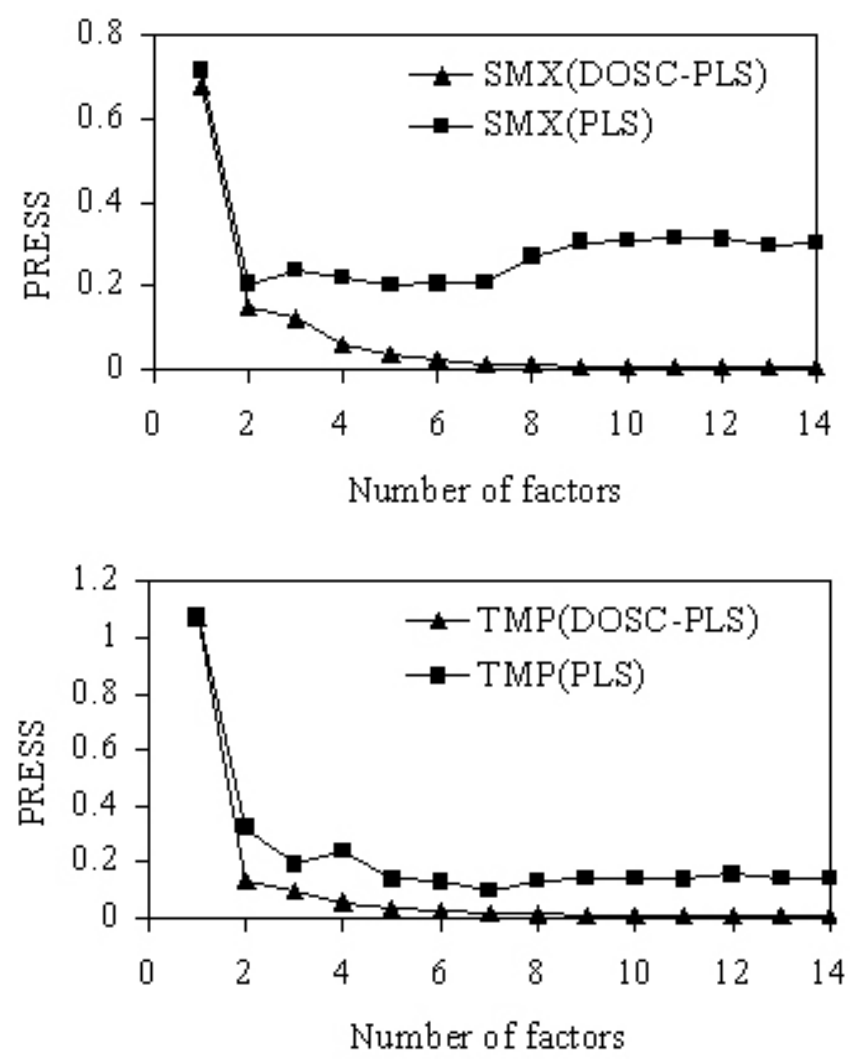
PLS.

Figure 2. Plots of PRESS versus number of factors by PLS and DOSC-

\section{Statistical parameters}

Four general statistical parameters were selected to evaluate the prediction ability of the constructed model. These parameters are correlation coefficient $\left(\mathrm{R}^{2}\right)$, root mean square error of prediction (RMSEP), relative standard error of prediction (RSEP) and mean absolute error (MAE). These parameters are calculated as follows:

$$
\begin{aligned}
& R M S E P=\sqrt{\frac{\sum_{i=1}^{n}\left(y_{\text {pred }}-y_{o b s}\right)^{2}}{n}} \\
& \operatorname{RSEP}(\%)=100 \times \sqrt{\frac{\sum_{i=1}^{n}\left(y_{\text {pred }}-y_{o b s}\right)^{2}}{\sum_{i=1}^{n}\left(y_{o b s}\right)^{2}}} \\
& \operatorname{MAE}(\%)=\frac{100}{n} \times \sqrt{\sum_{i=1}^{n}\left|\left(y_{\text {pred }}-y_{o b s}\right)\right|}
\end{aligned}
$$

Where $y_{\text {pred }}$ is the predicted concentration in the sample, $y_{\text {obs }}$ is the observed value of the concentration in the sample and $n$ is the number of samples in the validation set. The values for RMSEP, RSEP, MAE and $\mathrm{R}^{2}$ are given in Table 3.
Table 3. Statistical parameters of the optimized matrix using the PLS and DOSC-PLS.

\begin{tabular}{|l|l|l|l|l|l|}
\hline Component & NPC a & PRESS & RMSEP & RSEP (\%) & MAE(\%) \\
\hline Sulfamethoxazole $^{\mathrm{b}}$ & 3 & 0.1239 & 0.0221193 & 0.329735 & 5.654398 \\
\hline Trimethoprim $^{\mathrm{b}}$ & 3 & 0.0986 & 0.0236333 & 0.765090 & 5.876034 \\
\hline Sulfamethoxazole $^{\mathrm{c}}$ & 4 & 0.2207 & 0.170137 & 2.536255 & 15.9138 \\
\hline Trimethoprim $^{\mathrm{c}}$ & 3 & 0.1935 & 0.0166038 & 5.375223 & 14.7328 \\
\hline
\end{tabular}

${ }^{a}$ Number of principal component

${ }^{\mathrm{b}}$ Using DOSC-PLS

${ }^{c}$ Using PLS
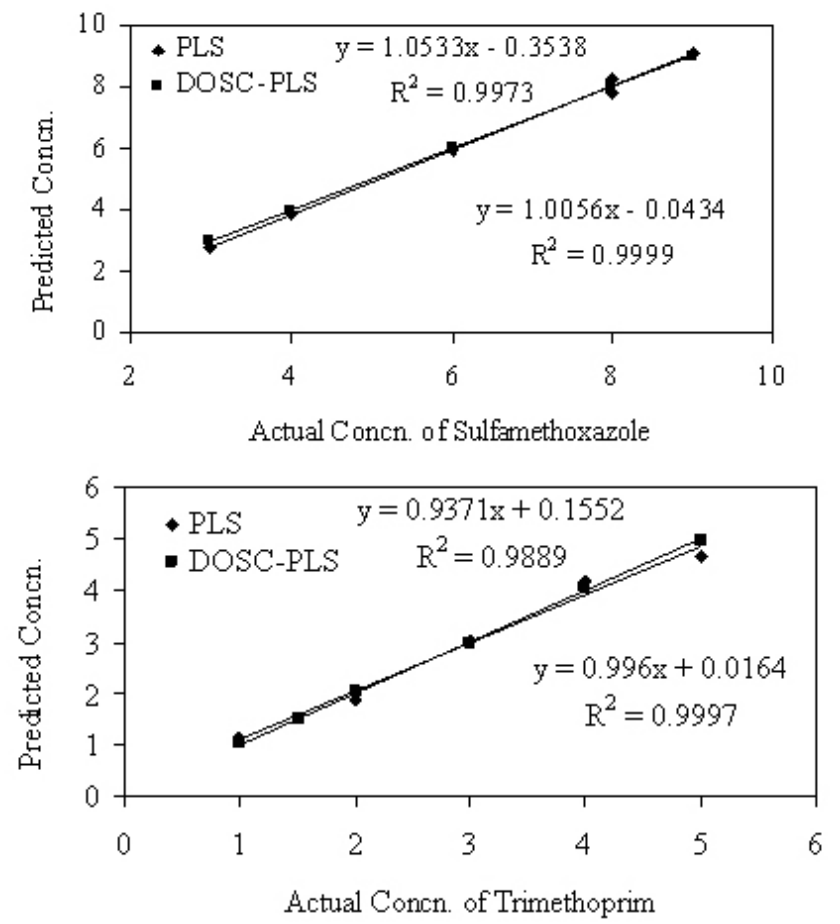

Figure 3. Plots of predicted concentration versus actual concentration for sulfamethoxazole and trimethoprim by PLS and DOSC-PLS $\left(\mu \mathrm{gmL}^{-1}\right)$. Determination of sulfamethoxazole and trimethoprim in real samples

In order to test the applicability and matrix interferences of the proposed method to the analysis of real samples, the method was applied in a variety of situations. For this purpose, diverse spiked samples and reference materials were analyzed. Table 4 shows the results obtained for real matrix samples. Therefore, the DOSC-PLS model is able to predict the concentrations of each sulfamethoxazole and trimethoprim in the real matrix sample.

\section{Study of foreign species}

In this study, other foreign species were added to the sample solution (containing both analytes) and their effects on the absorbance signals were investigated. Results are given in Table 5. The study was carried out with 200 and $40 \mu \mathrm{g} \mathrm{ml}^{-1}$ of SMX and TMP, respectively. Potential interfering species tested were among those usually accompanying the analytes in pharmaceutical preparations. The effect of each species was considered as an interference when caused a relative error in the signal of $\pm 3 \%$ in comparison with the signal obtained for each analyte in absence of the species, respectively. Some of the tested species did not cause interference even at the highest tested level because they are not sorbed on the solid sensing supports (e.g. lactose and saccharose). There is an evident difference between the tolerance values to the presence of foreign species in the determination of both compounds, as in all the cases 
the tolerance level is much lower for the determination of SMX than for TMP. It can be due to the weak retention of SMX on the solid support and so, to its determination practically in the interstitial solution between the beads of it. While TMP is strongly retained on the resin and so separated from the matrix (so increasing the selectivity of its determination), SMX is determined practically in homogeneous solution in the presence of other species that are not retained on the solid support owing to its neutral or anionic character at the working $\mathrm{pH}$ value.

Table 4. DOSC-PLS results applied on the real matrix samples.

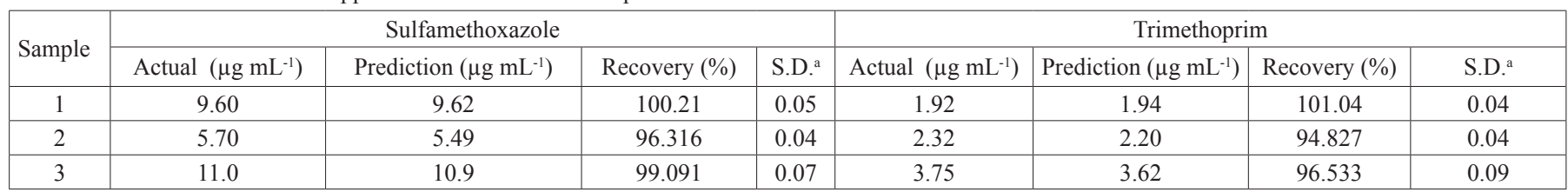

${ }^{a}$ Relative standard of deviation $(n=3)$

Table 5. Study of interferences $[\mathrm{SMX}]=200 \mu \mathrm{gml}^{-1}$; $[\mathrm{TMP}]=40 \mu \mathrm{gml}^{-1}$

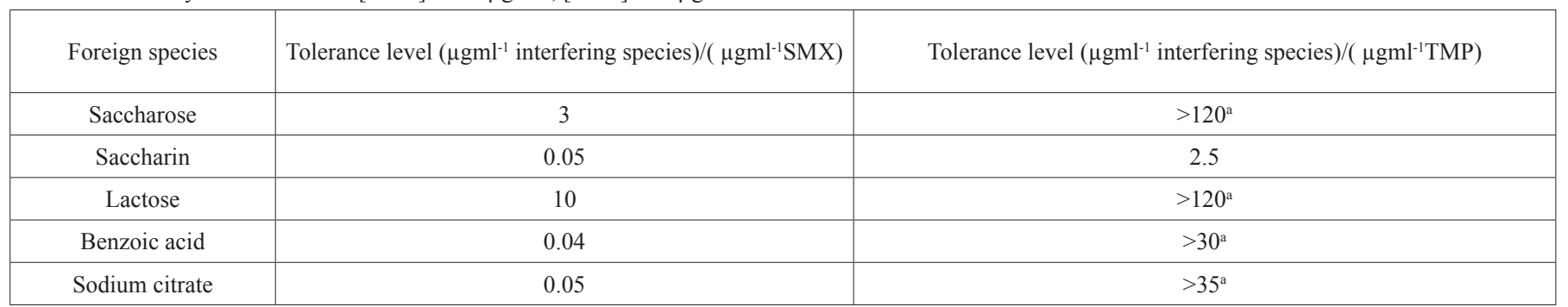

${ }^{a}$ Maximum ratio tested.

\section{CONCLUSION}

The simultaneous determination of sulfamethoxazole and trimethoprim mixtures by using spectrophotometric method is a difficult problem in analytical chemistry, due to spectral interferences, In order to overcome drawback, PLS and DOSC-PLS multivariate calibration approaches were applied and compared. Analysis of the results for secondary mixtures showed that the use of PLS leads to significantly less-accurate prediction. The predicted values are obtained by the application of DOSC-PLS model for absorbance data show the high prediction ability of the DOSC-PLS method. The good agreement clearly demonstrates the utility of this procedure for the simultaneous determination of sulfamethoxazole and trimethoprim in synthetic mixtures and pharmaceutical preparation.

\section{REFERENCES}

1. F.A.Mohamed, A.I.Mohamed, S.R.El-Shabouri, J. Pharm. Biomed. Anal.,6, 175, (1988).

2. P.Nagaraja, K.R.Sunitha, R.A. Vasantha, H.S. Yathirajan, Eur. J. Pharm. Biopharm., 53, 187,(2002).

3. A.Muñoz de la Pena, F.Salinas, I. Durán-Merás, M. Dolores Moreno, Anal. Lett., 27, 1893, (1994).

4. J.F.Fernández-Sánchez, A.Segura-Carretero, J.M.Costa-Fernández, N. Bordel, R. Pereiro, C.Cruces-Blanco, A.Sanz-Medel, A.FernándezGutiérrez, Anal. Bioanal. Chem. 377, 614,(2003).

5. T.B.Vree, A.J.A.M.Van der ven, C.P.W.G.M.Verwey-van Wissen, E.W.J. van Ewijk-Beneken Kolmer, A.E.M., Swolfs, P.M. van Galen, H. Amatdjais-Groenen, J. Chromatogr. B: Biomed. Sci. Appl.,658, 327,(1994).

6. Y.M.Issa, A.L.El-Ansary, W.Selim, Anal. Lett.,31, 131,(1998).

7. S.Z.Qureshi, M.I.H.Helaleh, N.Rahman, R.M.A.Q.Jamhour, Anal. Chem., 357, 1005,(1997).

8. M.Aboudan, Y.M.Issa, A.F.Shoukry, J. Chem. Technol. Biotechnol., 61, 31,(1994).

9. M.A.Ahmed, M.M. Elbeshlawy, Anal. Lett., 28, 2123, (1995).

10. M.W.Hruska, R.F.Frye, J. Chromatogr. B: Analyt. Technol. Biomed. Life. Sci., 807, 301, (2004).

11. P.Nachilobe, J.O.Boison, R.M.Cassidy, A.C.E. Fesser, J. Chromatogr. Biomed. Appl., 616, 243, (1993).
12. G.Chen, Y.H.Tu, L.V. Allen, M. Jr., D.P.Wang, Int. J. Pharm., 46, 89 (1988).

13. U.Ernemann, D.Schirmer, A.Wöber, P. Langmann, K.Warzecha, R Joeres, H. Klinker, E. Richter, J. Chromatogr. B: Biomed. Sci. Appl.,528, 243,(1990).

14. C.Altesor, P.Corbi, I.Dol, M.Knochen, Analyst.,118, 1549, (1993).

15. C.K.Markopoulou, E.T.Malliou, J.E.Koundourellis, Il Farmaco.,59, 627, (2004).

16. L.López-Martínez, P.L. López-de-Alba, L.M. de-León-Rodríguez, M.L.Yepez-Murrieta, J. Pharm. Biomed. Anal.,30, 77, (2002).

17. R.Gochin, I. Kanfer, J.M.Haigh, J. chromatogr. B: Biomed. Sci. Appl.,223, 139, (1981).

18. A.Niazi, M.Goodarzi, Spectrochim. Acta. A.,69, 1165, (2008).

19. M.Goodarzi, T.Goodarzi, N.Ghasemi, Ann. Chim.,97, 303, (2007).

20. G.Musmarra, D.F. Condorelli, S.Scire, A.S. Costa, Biochem. Pharmacol.,62, 547,(2001).

21. M.Lapinsh, P.Prusis, A.Gutcaits, T.Lundstedt, J.E.S. Wikberg, Biochim. Biophys. Acta., 1525, 180, (2001).

22. M.Jesús Gómez González, R.Olga Domínguez, M.Julia Arcos Martínez, Talanta.,68, 67,(2005).

23. M.Jalali-Heravi, S. Masoum, P.Shahbazikhah, J. Magn. Reson.,171, 176, (2004).

24. M.N.Taib, R.Andres, R. Narayanaswamy, Anal. Chim. Acta.,330, 31,(1996).

25. M.L.Luis, J.M.G. Fraga, F. Jiménez, A.I. Jiménez, J.J.Arias, Talanta.,53, $761,(2001)$.

26. S.Wold, H. Antti, F. Lindgren, J. Ohman, Chem. Intel. Lab. Syst.,44, $175,(1998)$

27. J.A.Westerhuis, S.D.Jong, A.K. Smilde, Chem. Intel. Lab. Syst.,56, $13,(2001)$

28. J.A. Cornel, Experimental with Mixtures, Wiley, New York, 1981.

29. J.C. Miller, J.N. Miller, Significance tests. In Statistics in Analytical Chemistry, third ed., Ellis Horwood, Chichester, 1993 (Chapter 3).

30. MATLAB 6.5, The Math Works Inc, Natick, MA.

31. G.Granero, C.Garnero, M.Longhi, J. Pharm. Biomed. Anal.,29, 51, (2002).

32. D.M.Haaland, E.V.Thomas, Anal. Chem.,60, 1193, (1988). 